\title{
Personalized Immunology in Cancer: Paving the Way Towards a Better Quality of Life
}

\author{
Abdolreza Esmaeilzadeh ${ }^{1,2,3, *}$, EInaz Khosh ${ }^{4}$ \\ ${ }^{1}$ Department of Immunology, School of Medicine, Zanjan University of Medical \\ Sciences, Zanjan, Iran \\ ${ }^{2}$ Cancer Gene Therapy Research Center (CGRC), Zanjan University of Medi- \\ cal Science, Zanjan, Iran \\ ${ }^{3}$ Immunotherapy Research and Technology Groups, Zanjan University of Medi- \\ cal Sciences, Zanjan, Iran \\ ${ }^{4}$ School of Medicine, Zanjan University of Medical Science, Zanjan, Iran \\ *Corresponding author: Abdolreza Esmaeilzadeh, Associate Professor, Department \\ of Immunology, School of Medicine, Zanjan University of Medical Sciences, Zanjan, \\ Iran.Tel: +982433440301; Fax:+982433449553; E-mail:a46reza@zums.ac.ir
}

DOI: $10.30699 / \mathrm{mci} \cdot 5 \cdot 1.441-1$

Submitted: 12 July 2020

Revised: 19 October 2020

Accepted: 31 October 2020

e-Published: 1 December 2020

\section{Keywords:}

Precision Medicine

Immunotherapy, Adoptive

Patient-Specific Modeling

Cancer Vaccines
Conventionally, in specific diseases, patients receive similar therapies; relying on a "one size fits all" approach. The discovery of P5 (Predictive, Preventive, Personalized, Participatory, and psycho-cognitive) improves personalized diagnosis, treatment, and prognosis. Considering the high prevalence, mortality rate, and complexity of cancers, there is a critical necessity to choose specific and individualized therapies in each case. In this point of view, immunology has a vital role in the biological variability of the patient-to-patient system and tumor microenvironment heterogeneity. Thus, it is required to improve personalized immunology in various diseases and cancers as well. Notably, personalized immunology will develop personalized cancer treatments and improve clinical outcomes. In this study, we proposed to review the essential roles of personalized immunology in cancer personalized therapies. Also, we can suggest the transformation of P5 medicine into P6 medicine, with the sixth P standing for personalized immunology.

(C) 2021. Multidisciplinary Cancer Investigation

\section{INTRODUCTION}

A conventional treating method in medicine is based on signs and symptoms. A so-called term "one size fits all" relates to this approach, and it does not take the patients into account individually [1]. As a consequence, the $\mathrm{P} 4$ medicine theory was proposed in 2010; containing "Predictive, Preventive, Personalized, and Participatory" components [2]. Gorini et al., transformed P4 medicine to P5 medicine in 2011. They announced the fifth $P$, which was the psycho-cognitive dimension, to consider the behavioral features of individuals, such as prevention activities, interaction with healthcare workers, choosing a therapy among different treatments, and adherent to their selected treatment process [3]. The most examined field for personalized medicine is cancer. Common cancer treatment methods are chemotherapy, radiotherapy, and conservative surgery. It is worth noting that each cancer requires specific treatment. Hence, it is essential to be familiar with each tumor microenvironment, genetic mutations, immunological abnormalities, and signaling pathways to achieve the most 
appropriate therapies for cancerous patients, separately. It seems that the mutations in tumors would not result in the same therapeutic outcomes in different patients with various genetical abnormalities. All of the patient-specific factors, such as inheritance, past medical histories, smoking, clinical and molecular heterogeneity, and various immune system will influence the dynamic of the tumor, so we cannot anticipate the successful clinical improvement in all patients. However, the application of personalized medicine and personalized immunology is more complicated for particular conditions, such as malignancies. The modern personalized cancer therapies are almost based on common genetic mutations and targeting conventional biomarkers in types of cancers. An example of this approach is imatinib, which is used to treat chronic myeloid leukemia (CML) with the BCR-ABL translocation [4]. The latest advances in technologies and bioinformatics could discover novel targeting biomarkers based on immunogenetical abnormalities in tumor cell lines and immunological characteristics of the patients. It is substantial for scientists to be accustomed to immunological variability in each patient, to obtain the most accurate therapy. Herein, according to the differences in immunology and genetics in patients, there will be a need to design personalized cancer treatment methods to improve the clinical outcomes. Hence, in this article, we proposed to define the effects of personalized immunology on cancer treatment.

\section{P4 Medicine}

All the individual-specific data would be achievable in the next ten years for the research activities to improve the predictive section of the $\mathrm{P} 4$ theory. Indeed, preventive medicine indicates that the focus of researches should be on diseases, not medicine. To evaluate the health state of each case, all the people should have a regular check-up for preventing specific disease progression. Personalized medicine believes that everyone is different in genome sequences and the environment. So monitoring for heath-being and wellness should be performed individually based on patient-specific documents. The well-established opinion of personalized medicine is administered in diabetic patients in whom the dosage of insulin or their regimen could be set regarding the measured blood glucose level. Additionally, the process of patient management is better to be patient-derived in participatory medicine. In short, the P4 medicine considers each patient separately to achieve the appropriate diagnosis and proper treatment choices. The P4 medicine and system biology endeavor to create novel advancements to overcome disease complexities, develop the health state, and decrease the costs of healthcare for individuals [5-8].

\section{Personalized/Precision Medicine}

One of the recent scopes in patients' management is personalized medicine which is being extended. The latest progression in personalized fields demonstrated that personalized medicine and precision medicine are equivalent and can be administered interchangeably; however, there are some trivial differences. The precision medicine method categorizes the population into different subsets; each subpopulation would be dissimilar regarding the predisposition to particular diseases. The subpopulation selection would be feasible by considering the variability of the patients and the mechanism of the diseases. It should be pointed out that the "personomics" term is essential to dedicate the specialized health care facilities for each individual [9]. Also, we should consider that all the personalized methods have to be more implementable; based on time schedules and costeffectiveness for the cases [10]. Every single case is different in regards to its signs and symptoms; also, patients vary in style of living, environment, and genes. Furthermore, the studies have revealed that the response of the patients to certain drugs is not only at a different level in all individuals, but also it may have disadvantages in some patients. Researches represented that the progression of the personalized drug screening (PDS) approach could be able to target specific molecules in each patient. In addition, PDS can define targeted therapies even in diseases without distinct treatments. Moreover, the combination of PDS, genetics, and immunology would develop personalized therapeutic strategies $[11,12]$. For instance, the response to antiretroviral therapy is not at the same level in HIV/AIDS patients. The documents have indicated the association between CCR5 gene polymorphisms and the natural resistance of the patients to HIV. Thus, recognizing the CCR5 gene polymorphisms, and genetic profile of the HIV/AIDS patients could help the scientists towards appropriate antiretroviral therapies. In this 
way, the patients that are homozygous for the CCR5 gene are naturally resistant to HIV penetration, and HIV could not be able to enter the host immune cells $[13,14]$. Researches have represented the HIV remission in "Berlin patient" and "London patient" after transplanting the homozygous CCR5 delta $32 \mathrm{bp}$ allogeneic hematopoietic stem cell to them $[15,16]$. These documents are indicating the stunning roles of CCR5 gene polymorphisms in HIV immunopathogenesis. There is a requirement for the genome and molecular analysis to determine personalized biomarkers associated with endotype (a subtype of a condition which is defined by a distinct functional or pathobiological mechanism), genotype, the complexity of diseases, and specific "omics" information. Although conventional immunology implicates in basic and clinical aspects, personalized immunology is based on targetting downstream practical molecular factors, extending personalized genetic biomarkers, and stratifying cases; based on "omics" data [17].

\section{Immune-Oncology}

Immune-related disorders, such as auto-immune diseases, infectious diseases, inflammatory conditions, atopic dermatitis, and psoriasis are being discussed in personalized immunology strategy [18, 19]. Furthermore, immune-oncology and personalized cancer medicine are novel issues in recent topics. Besides tumor proliferation, angiogenesis, and metastasis, some microscopic changes such as chromosomal abnormalities and genetic mutations cause tumor progression, so understanding the precise mechanisms of genetics and immunological signaling pathways of tumors would guide the physicians toward developing novel targeted therapies to destroy tumor cells without affecting adjacent and healthy tissues in each tumor microenvironment (TME) [17, 20].

\section{Tumor Microenvironment}

TME is a complex cell-cell interaction that includes various types of cells; some of them are crucial for promoting the immune response against tumor proliferation, and some of the cells are acting as immunosuppressors. This situation provides a profound inter and intratumor heterogeneity, so the molecular discovery of each TME could improve the field of cancer treatment. Thus, personalized immunology would help to improve clinical outcomes. The main goal of personalized treatment is targeting the proper molecules according to the patient-specific "omics" data. Thereby, there is an essential need to analyze the "omics" approach in each case including; immunoproteomics, immunogenomics, immune metabolomics, and immune personomics that are expressed in the personalized immunology. Taken together, the omics data analysis and whole-genome sequencing will assist the scientists to identify the different TMEs in every single case and also to discover personalized neo-antigen cancer vaccines. Likewise, single-cell DNA sequencing such as cancer stem cells and circulating cancer cells (CTCs) have the potentials to determine the TME heterogeneity and tumor invasion. Generally, the latest technologies could provide an approach to discover different omics data from a single cell of TME. Consequently, this method could enable researchers to determine the inter and intratumor heterogeneity, neo-antigen mutations, unusual signaling pathways, and all the immunopathological conditions that have occurred in individuals [21, 22]. Considerably, there are some cancer immunotherapy strategies including the blockage of immune-checkpoints, cancer vaccines, and adoptive cell therapy. Indeed, different TMEs can inhibit the anti-tumor immune responses that are various in each patient. Thus, further investigations are required to determine the anti-tumor immune responses toward designing more accurate personalized cancer immunotherapy approaches.

\section{Immune-Checkpoint Inhibitors}

The blockage of immune-checkpoints is the application of immunology in oncology. Studies have demonstrated that interaction between CD80/86 on DCs and the cytotoxic T-lymphocyteassociated protein 4 (CTLA-4) on T cells leads to some inhibitory signals to the $\mathrm{T}$ cells. Moreover, the interaction between the programmed cell death protein 1 (PD-1) on activated T cells and its ligand (PD-L1) on tumor cells impairs the function and the proliferation of the $\mathrm{T}$ cells. Nowadays, one of the standard treatments in cancers is blocking the abovementioned immune-checkpoint inhibitors, such as PD-1, PD-L1, and CTLA-4 by monoclonal antibodies (mAbs). Notably, in 2011, an antiCTLA-4 mAb (Ipilimumab) was approved by the FDA for treating melanoma [23]. Additionally, an anti-PD-1 mAb (Nivolumab) had demonstrated 
appropriate responses in treating some solid cancers [24]. Nivolumab was approved in Japan for treating lung cancer and malignant melanoma. It is expected that the abovementioned approvals would happen for other types of cancers, such as lymphoma, bladder cancer, triple-negative breast cancer, and renal cell carcinoma (RCC). It should be noted that immune-checkpoints are different in each individual and TMEs; therefore, identifying the patients' genotypes and phenotypes would be practical. The cancer vaccines would act appropriately if they target the specific molecules in each case with their exclusive TME. Accordingly, targeting immunecheckpoint inhibitors could be a precious method in personalized immunotherapy [25].

\section{Cancer Vaccine}

The documents have shown that the investigations for finding more efficient cancer vaccines are going on [26]. One of the recent discoveries in the cancer vaccine is the usage of dendritic cells. To improve the efficacy of dendritic cell-based vaccines, checking the subsets of dendritic cells in individuals will be required $[19,27]$. It is worth noting that dendritic cell-based vaccines are the most powerful strategies in personalized immunotherapy which could be followed by personalized responses. Other studies have found cancer neoantigens that could be targeted by immunotherapy methods such as cancer vaccines. Cancer neoantigens are the results of mutations in somatic cells in each patient. Accordingly, increasing the knowledge about cancer neoantigens in each case is needed [20, 28]. Indeed, Exosomes are cell-free mediators of the immune system which act for intercellular connection. The administration of allogeneic exosome cancer vaccines would lead to a roadmap toward personalized immunology therapies [29, 30]. Some other studies demonstrated the therapeutic potentials of autologous heat shock protein (HSP) for cancer vaccines. HSPs can bind to the peptides and transporting them through the cells [25]. For cancer vaccine production, HSP-peptide complexes are isolated from resected tumors. In 2008, HSP-peptide complex-96 (HSPPC-96) was approved in Russia for early-stage kidney cancers [31]. Altogether, analyzing the patient-to-patient immune system variation would help scientists to discover efficacious personalized cancer vaccines.

\section{Adoptive Cell Therapy}

One of the latest approaches in personalized immunotherapy is adoptive cell therapy (ACT) with the usage of tumor-infiltrating lymphocytes or $\mathrm{T}$ cells expressing chimeric antigen receptor (CAR). Equipped T cells with CAR (CAR T cells) are one of the novel immunotherapeutic approaches in personalized cancer treatment. In this strategy, the T cells that are obtained from each patient peripheral blood mononuclear cells (PBMCs), are genetically modified to express CAR on their cell surface. CAR is designed to target specific antigens in each TME. Of note, this approach has led to complete remission in some B cell lymphoid malignancies; however, many clinical trials in this field are going on to achieve prosperities in cancer therapeutic strategies [32-35].

\section{Biomarkers}

One of the most complicated fields in personalized immunology is various types of cancer. Notably, personalized cancer medicine would be effective in detecting more suitable biomarkers for immunotherapy usage and improving the clinical outcomes. Since finding appropriate biomarkers in the individual patient has a substantial role in personalized therapies. So, detecting the biomarkers with high efficiency is the fundamental approach in personalized cancer medicine [36]. The examples of administrating biomarkers in cancers are as follows: the usage of cancer antigen 19-9 (CA19-9) has shown to be useful in predicting the prognosis and recurrence of the tumor with $80 \%$ sensitivity and $90 \%$ specificity in pancreatic cancer models [37, 38]. Some of the other detectable markers in specific conditions are the application of prostate-specific antigen (PSA) in prostate cancer [39], carcinoembryonic antigen (CEA) in colorectal cancer [40], and CD20 in relapsed and/ or refractory follicular lymphoma [41]. Moreover, we should consider that the measured levels of the proteomics will be influenced by the age of the patients, concomitant medical conditions, smoking, nationality, and inheritance $[42,43]$. It seems that the simultaneous utilization of the biomarker analysis and individual immuno-genetical and pharmacogenetics analysis would be a considerable outlook in personalized cancer therapies (Figure 1). 


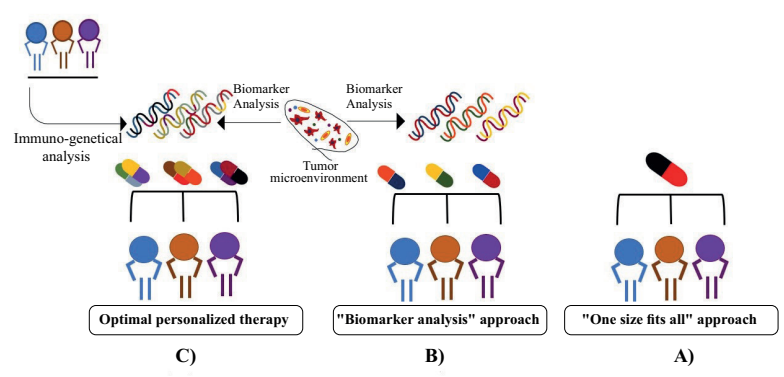

Figure 1: The Perspective on the Previous, Recent, and Personalized Immunology Treatment in Cancer

A) One size fits all approach: in this approach, all the patients receive similar drugs; B) Biomarker analysis approach: in this approach, the patients receive personalized drugs based on molecular analysis of the tumor microenvironment; C) Optimal personalized therapy: in this approach, patients receive the most accurate and specific therapies according to biomarker analysis of the tumor microenvironment and immune-genetical evaluation of the patients.

\section{Tools}

To date, scientists and clinicians have administered microarray analysis and next-generation sequencing (NGS) to determine the tumor cell progression, the exclusive inter and intratumor heterogeneity, the concomitant genetics, and epigenetics abnormalities in every single case. The mentioned tools could make a chance to promote personalized immunology in the diagnosis, prognosis, and treatment of the cancers $[44,45]$.

\section{The Potential of Personalized Cancer Medicine and Its Limitations}

The latest advancements in cancer field research have discovered the particular molecular pathways in each TMEs which could facilitate and expand the specific targeted therapies in each cancer. The ongoing studies on the genetics, immunological, and molecular pathways in the TME have led the researchers and scientists toward personalized cancer medicine. The targeted therapies will improve the outcomes of cancer treatment, patient survival, and quality of life. Studies have revealed that the specimens from different parts of the same tumor in patients have shown that some mutations in signaling pathways are identical, but some of them are unique in each microenvironment. So the exclusive mutations that influence the proliferation or the survival of the tumor cells would be advantageous in personalized cancer medicine. However, there are some obstacles in the progression process of personalized cancer medicine. Inter and intratumor heterogeneity is the most challenging pitfall of cancer treatment which can affect the selection of the targeted molecules. Another problem in targeted therapy is inadequate tumor samples for determining the appropriate targeted therapy. Since the targeted molecules should be expressed in the majority of tumor cells and have a role in the proliferation of the cancer cells, it is so difficult to recognize the confined genes, cellular, and molecular markers, signaling pathways, and specific proteomics based on "omics" data analysis to be targeted by personalized immune gene therapy approach. Likewise, we should consider that the targeted metabolites may be unsteady, irreproducible, and unreliable. High economic costs, disunited, unregulated, non-digitalized, and continuously increasing data are other restrictions of personalized immunology applications. Indeed, there is insufficient information about mechanisms of the disease as well as various immune cells' properties in exposure to environmental factors. Hence, the evaluation of the molecular characteristics in individual tumors and overcoming the existing limitations would assist the scientists to enhance personalized immunology in cancer treatment $[17,46]$.

\section{CONCLUSION}

Generally, the administration of immunotherapy is applied in the treatment of various human malignancies, auto-immune, infectious diseases, and inflammatory skin disorders. However, there are some boundaries in the usage of immunotherapy as a treatment method. Hence, there is a need to comprehend the whole genetics and epigenetics issues of individuals to go beyond the limitations. As mentioned earlier, there are some challenges and difficulties in the immunology field, in which the response of some patients to immunotherapy is low. The different responses of patients can be related to their specific TMEs which are expressing particular tumor antigens. Taken together, more studies and researches are required to improve personalized immunology in individuals. Personalized immunology will assess the scientists to distinguish patientspecific molecular mechanisms in particular diseases. Categorizing the population watchfully would be so beneficial towards personalized immunology and personalized treatment. Personalized immunology is a precious chance to promote human wellbeing; thus, the advantages of this approach will guarantee personalized treatment and our healthiness, 
accordingly. Furthermore, one of the points of view in the improvement of personalized medicine could be suggesting personalized immunology as the sixth $\mathrm{P}$ of the previous theories (Figure 2).

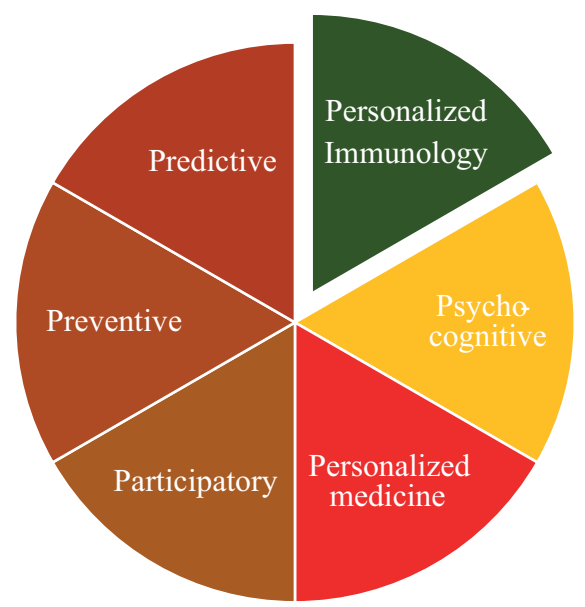

Figure 2: Personalized immunology as the sixth $\mathrm{P}$ of the previous P5 theory

Up to now, many therapeutic trials have been designed in mouse and human models to recognize and envision patient-to-patient system biology variability, as well as inter and intratumor heterogeneity. These trials promote personalized immunology toward more successful therapies in various types of malignancies. Notably, personalized cancer medicine will have a higher price; however, maybe it is the only way to escape from unnecessary treatments, inappropriate costs, and to achieve better survival and quality of life.

\section{ACKNOWLEDGMENTS}

We express our special thanks to all authors who have worked in the personalized medicine and personalized immunology fields, but we cannot reference their precious studies due to the accessibility limitations.

\section{CONFLICT OF INTEREST}

The authors declared no conflict of interest.

\section{ETHICS APPROVAL}

This review article contains no ethical consideration.

\section{REFERENCES}

1. Chung KF. Personalised medicine in asthma: time for action: Number 1 in the Series "Personalised medicine in respiratory diseases" Edited by Renaud Louis and Nicolas Roche. Eur Respir Rev. 2017;26(145):170064. DOI:
10.1183/16000617.0064-2017 PMID: 28954768.

2. Auffray C, Charron D, Hood L. Predictive, preventive, personalized and participatory medicine: back to the future. Genome Med. 2010;2(8):57. DOI: 10.1186/gm178 PMID: 20804580 .

3. Gorini A, Pravettoni G. P5 medicine: a plus for a personalized approach to oncology. Nat Rev Clin Oncol. 2011;8(7):444. DOI: 10.1038/nrclinonc.2010.227-c1 PMID: 21629214.

4. Druker BJ, Talpaz M, Resta DJ, Peng B, Buchdunger E, Ford JM, et al. Efficacy and safety of a specific inhibitor of the BCR-ABL tyrosine kinase in chronic myeloid leukemia. N Engl J Med. 2001;344(14):1031-7. DOI: 10.1056/ NEJM200104053441401 PMID: 11287972.

5. Hood L. Systems biology and p4 medicine: past, present, and future. Rambam Maimonides Med J. 2013;4(2):e0012. DOI: $10.5041 /$ RMMJ.10112 PMID: 23908862.

6. Hood L, Friend SH. Predictive, personalized, preventive, participatory (P4) cancer medicine. Nat Rev Clin Oncol. 2011;8(3):184-7. DOI: 10.1038/nrclinonc.2010.227 PMID: 21364692.

7. Flores M, Glusman G, Brogaard K, Price ND, Hood L. P4 medicine: how systems medicine will transform the healthcare sector and society. Pers Med. 2013;10(6):565-76. DOI: $10.2217 /$ pme.13.57 PMID: 25342952.

8. Sobradillo P, Pozo F, Agusti A. P4 medicine: the future around the corner. Arch Bronconeumol. 2011;47(1):35-40. DOI: 10.1016/S1579-2129(11)70006-4 PMID: 21190770.

9. Ziegelstein RC. Personomics: The missing link in the evolution from precision medicine to personalized medicine. J Pers Med. 2017;7(4):11. DOI: 10.3390/jpm7040011 PMID: 29035320.

10. Mathur L, Ballinger M, Utharala R, Merten CA. Microfluidics as an Enabling Technology for Personalized Cancer Therapy. Small. 2019;16(9):e1904321. DOI: 10.1002/ smll.201904321 PMID: 31747127.

11. Braig Z. Personalized medicine: From diagnostic to adaptive. Biomed J. 2019. DOI: 10.1016/j.bj.2019.05.004.

12. Gorshkov K, Chen CZ, Marshall RE, Mihatov N, Choi $\mathrm{Y}$, Nguyen D-T, et al. Advancing precision medicine with personalized drug screening. Drug Discov Today. 2019;24(1):272-8. DOI: 10.1016/j.drudis.2018.08.010 PMID: 30125678.

13. Esmaeilzadeh A, Farshbaf A. Novel approaches based on autologous stem cell engineering and gene-modification; evidence for the Cure of HIV/AIDS. J Genet Syndr Gene Ther. 2015;6(2):3-4. DOI: 10.4172/2157-7412.1000e130.

14. Esmaeilzadeh A, Farshbaf A, Erfanmanesh M. Autologous Hematopoietic Stem Cells transplantation and genetic modification of CCR $5 \mathrm{~m} 303 / \mathrm{m} 303$ mutant patient for HIV/AIDS. Med Hypotheses. 2015;84(3):216-8. DOI: 10.1016/j.mehy.2014.12.027 PMID: 25613565.

15. Hutter G, Nowak D, Mossner M, Ganepola S, Müßig A, Allers K, et al. Long-term control of HIV by CCR5 Delta32/Delta32 stem-cell transplantation. N Engl J Med. 2009;360(7):692-8. DOI: 10.1056/NEJMoa0802905 
PMID: 19213682.

16. Gupta RK, Peppa D, Hill AL, Gálvez C, Salgado M, Pace $\mathrm{M}$, et al. Evidence for HIV-1 cure after CCR5 $\Delta 32 / \Delta 32$ allogeneic haemopoietic stem-cell transplantation 30 months post analytical treatment interruption: a case report. Lancet HIV. 2020;7(5):e340-e7. DOI: 10.1016/S23523018(20)30069-2 PMID: 32169158.

17. Delhalle S, Bode SF, Balling R, Ollert M, He FQ. A roadmap towards personalized immunology. NPJ Syst Biol Appl. 2018;4(1):1-14. DOI: 10.1038/s41540-017-0045-9 PMID: 29423275

18. Canonica GW, Ferrando M, Baiardini I, Puggioni F, Racca F, Passalacqua G, et al. Asthma: personalized and precision medicine. Curr Opin Allergy Clin Immunol. 2018;18(1):51-8. DOI: 10.1097/ACI.0000000000000416 PMID: 29189350.

19. Litman T. Personalized medicine - concepts, technologies, and applications in inflammatory skin diseases. APMIS. 2019;127(5):386-424. DOI: 10.1111/apm.12934 PMID: 31124204.

20. Mandal R, Chan TA. Personalized oncology meets immunology: the path toward precision immunotherapy. Cancer Discov. 2016;6(7):703-13. DOI: 10.1158/2159-8290.CD16-0146 PMID: 27107038.

21. Finotello F, Eduati F. Multi-omics profiling of the tumor microenvironment: paving the way to precision immuno-oncology. Front Oncol. 2018;8:430. DOI: 10.3389/ fonc.2018.00430 PMID: 30345255.

22. Navin NE. The first five years of single-cell cancer genomics and beyond. Genome Res. 2015;25(10):1499-507. DOI: 10.1101/gr.191098.115 PMID: 26430160.

23. Hodi FS, O'Day SJ, McDermott DF, Weber RW, Sosman JA, Haanen JB, et al. Improved survival with ipilimum$\mathrm{ab}$ in patients with metastatic melanoma. N Engl J Med. 2010;363(8):711-23. DOI: 10.1056/NEJMoa1003466 PMID: 20525992.

24. Topalian SL, Hodi FS, Brahmer JR, Gettinger SN, Smith DC, McDermott DF, et al. Safety, activity, and immune correlates of anti-PD-1 antibody in cancer. N Engl J Med. 2012;366(26):2443-54. DOI: 10.1056/NEJMoa1200690 PMID: 22658127.

25. Kakimi K, Karasaki T, Matsushita H, Sugie T. Advances in personalized cancer immunotherapy. Breast Cancer. 2017;24(1):16-24. DOI: 10.1007/s12282-016-0688-1 PMID: 27000871.

26. Jacob M, Lopata AL, Dasouki M, Abdel Rahman AM. Metabolomics toward personalized medicine. Mass Spectrom Rev. 2019;38(3):221-38. DOI: 10.1002/mas.21548 PMID: 29073341.

27. Mastelic-Gavillet B, Balint K, Boudousquie C, Gannon PO, Kandalaft LE. Personalized Dendritic Cell Vaccines-Recent Breakthroughs and Encouraging Clinical Results. Front Immunol. 2019;10:766. DOI: 10.3389/fimmu.2019.00766 PMID: 31031762.

28. Guo Y, Lei K, Tang L. Neoantigen vaccine delivery for personalized anticancer immunotherapy. Front Immunol.
2018;9:1499. DOI: 10.3389/fimmu.2018.01499 PMID: 30013560 .

29. Apostolopoulos V. Cancer Vaccines: Research and Applications. Cancers (Basel). 2019;11(8):1041. DOI: 10.3390/ cancers11081041 PMID: 31344788.

30. Samuel M, Gabrielsson S. Personalized medicine and back-allogeneic exosomes for cancer immunotherapy. J Intern Med. 2019. DOI: 10.1111/joim.12963 PMID: 31359504.

31. Srivastava PK. Immunotherapy of human cancer: lessons from mice. Nat Immunol. 2000;1(5):363-6. DOI: 10.1038/80795 PMID: 11062489.

32. Elahi R, Khosh E, Tahmasebi S, Esmaeilzadeh A. Immune cell hacking: challenges and clinical approaches to create smarter generations of chimeric antigen receptor T cells. Front Immunol. 2018;9:1717. DOI: 10.3389/fimmu.2018.01717 PMID: 30108584

33. Esmaeilzadeh A, Tahmasebi S, Athari SS. Chimeric antigen receptor-T cell therapy: Applications and challenges in treatment of allergy and asthma. Biomed Pharmacother. 2020;123:109685. DOI: 10.1016/j.biopha.2019.109685 PMID: 31862474

34. Tahmasebi S, Elahi R, Esmaeilzadeh A. Solid tumors challenges and new insights of car t cell engineering. Stem Cell Rev Rep. 2019;15(5):619-36. DOI: 10.1007/s12015-01909901-7 PMID: 31161552.

35. Tahmasebi S, Elahi R, Khosh E, Esmaeilzadeh A. Programmable and multi-targeted CARs: a new breakthrough in cancer CAR-T cell therapy. Clin Transl Oncol. 2020:[Epub ahead of print]. DOI: 10.1007/s12094-020-02490-9 PMID: 32997278

36. Li J, Wang D, Wang Y. IBI: Identification of Biomarker Genes of Individual Tumor Sample. Front Genet. 2019;10:1236. DOI: 10.3389/fgene.2019.01236 PMID: $\underline{31850079}$

37. Kim JE, Lee KT, Lee JK, Paik SW, Rhee JC, Choi KW. Clinical usefulness of carbohydrate antigen $19 \square 9$ as a screening test for pancreatic cancer in an asymptomatic population. J Gastroenterol Hepatol. 2004;19(2):1826. DOI: 10.1111/j.1440-1746.2004.03219.x PMID: 14731128

38. Steinberg W. The clinical utility of the CA 19-9 tumor-associated antigen. Am J Gastroenterol. 1990;85(4):350-5. PMID: 2183589.

39. Holdenrieder S, Pagliaro L, Morgenstern D, Dayyani F. Clinically meaningful use of blood tumor markers in oncology. Biomed Res Int. 2016;2016:9795269. DOI: 10.1155/2016/9795269 PMID: 28042579.

40. Ichiki AT, Krauss S, Israelsen KL, Sonoda T, Collmann R. Sequential Carcinoembryonic Antigen Levels. Oncology. 1981;38(1):27-30. DOI: 10.1159/000225516 PMID: $\underline{7443173}$

41. Hagenbeek A, Gadeberg O, Johnson P, Møller Pedersen L, Walewski J, Hellmann A, et al. First clinical use of ofatumumab, a novel fully human anti-CD20 monoclonal antibody in relapsed or refractory follicular lymphoma: results 
of a phase 1/2 trial. Blood. 2008;111(12):5486-95. DOI: 10.1182/blood-2007-10-117671 PMID: 18390837.

42. Coombes KR, Morris JS, Hu J, Edmonson SR, Baggerly KA. Serum proteomics profiling - a young technology begins to mature. Nat Biotechnol. 2005;23(3):291-2. DOI: 10.1038/nbt0305-291 PMID: 15765078.

43. Selleck MJ, Senthil M, Wall NR. Making meaningful clinical use of biomarkers. Biomark Insights. 2017;12:1177271917715236. DOI: 10.1177/1177271917715236 PMID: 28659713.

44. Liu L, So AY-L, Fan J-B. Analysis of cancer genomes through microarrays and next-generation sequencing. Transl Cancer Res. 2015;4(3):212-8. DOI: 10.3978/j. issn.2218-676X.2015.05.04.

45. Suwinski P, Ong C, Ling MH, Poh YM, Khan AM, Ong HS. Advancing personalized medicine through the application of whole exome sequencing and big data analytics. Front Genet. 2019;10:49. DOI: 10.3389/fgene.2019.00049 PMID: 30809243 .

46. Tannock IF, Hickman JA. Limits to personalized cancer medicine. N Engl J Med. 2016;375(13):1289-94. DOI: 10.1056/NEJMsb1607705 PMID: 27682039. 